\title{
Évolutions technologiques et évolution des systèmes de production
}

\section{Claude Béranger}

\section{(2) OpenEdition \\ 1 Journals}

\section{Édition électronique}

URL : http://journals.openedition.org/economierurale/2730

DOI : 10.4000/economierurale.2730

ISSN : 2105-2581

Éditeur

Société Française d'Économie Rurale (SFER)

\section{Édition imprimée}

Date de publication : 2 août 2005

Pagination : 86-90

ISSN : 0013-0559

\section{Référence électronique}

Claude Béranger, «Évolutions technologiques et évolution des systèmes de production », Économie rurale [En ligne], 288 | Juillet-août 2005, mis en ligne le 05 juillet 2009, consulté le 21 décembre 2020. URL : http://journals.openedition.org/economierurale/2730 ; DOI : https://doi.org/10.4000/ economierurale. 2730 


\section{Évolutions technologiques et évolution des systèmes de production}

\section{Claude BÉRANGER • Institut national de la recherche agronomique, Paris}

En introduction au débat sur l'évolution des systèmes de production agricoles face aux nouvelles exigences de la société, je voudrais réfléchir avec vous sur la manière dont les évolutions technologiques ont permis, entraîné ou croisé ces évolutions souhaitables des systèmes de production. Je ferai cette analyse critique à partir de ma longue expérience personnelle des questions de recherche appliquée dans le domaine de l'agriculture.

\section{L'évolution des conceptions}

Cette évolution des systèmes de production a d'abord conduit, à la fin des modèles simples et dominants, des paquets techniques universels, face à l'accroissement de la diversité des agricultures européennes, des systèmes de productions localisés, que l'élargissement de l'Europe ne fait que renforcer.

Si la science se dit universelle, les technologies agricoles ne le sont guère ; elles doivent s'adapter aux divers contextes, voire se renouveler, et surtout être situées et raisonnées au sein de systèmes de production variés. Et ce sont ces systèmes d'exploitation qui doivent s'ajuster aux demandes sociétales et à leurs variations dans l'espace et le temps. Ils réagissent par ailleurs sur les technologies et appellent assez souvent leur révision ou des innovations.

Ainsi, pour bâtir les systèmes souhaités, les progrès fulgurants de la génétique permettent certes des adaptations rapides et astucieuses, mais la biodiversité des ressources génétiques naturelles ou fabriquées par des millénaires de civilisations diverses est aussi une richesse essentielle dont la sauvegarde et la gestion sont devenues nécessaires.

Les performances recherchées ne sont pas celles de chaque technique mais celles du système global, en terme quantitatif et qualitatif, souvent difficiles à bien apprécier.

Le modèle de transfert linéaire, descendant de la science à la technique et à son application, se trouve progressivement (et souvent trop lentement) remplacé par un modèle interactif et circulaire qui met en jeu les différents acteurs impliqués dans et par le système.

\section{L'évolution des enjeux et des objectifs}

Il est maintenant généralement admis que les systèmes d'exploitation devaient intégrer différents enjeux et objectifs liés aux contextes actuels :

- Le temps et l'organisation du travail qu'impliquent l'agrandissement des exploitations et des troupeaux, la gestion de la complexité croissante des systèmes de production, les exigences de la multifonctionnalité de l'agriculture, la part

1. Claude Béranger est spécialiste de la production de viande bovine, l'élevage et de la nutrition des herbivores et, plus largement, des systèmes agraires et du développement agricole. Tout au long de sa carrière à l'INRA (1958-2001), en particulier lorsqu'il fut Directeur scientifique, il a accompagné le Développement agricole dans son adaptation au développement durable, au développement régional et dans la qualification des produits de terroir. 
croissante du travail administratif, la pluriactivité de plus en plus fréquente. Le sens du métier d'agriculteur s'en trouve profondément modifié.

- La réduction des coûts de production, des dépenses énergétiques qui conduisent en partie à une agriculture plus autonome.

- La réduction des diverses pollutions des milieux, engendrées par les activités agricoles.

- La maîtrise de la qualité au niveau des produits, mais aussi des ressources, des pratiques, des milieux concernés. On ne peut se satisfaire de quelques caractéristiques simples et faciles à mesurer.

- La participation de l'agriculture à la production d'énergies renouvelables, notamment par celle de biocarburants

- L'organisation des filières de produits et des marchés et les relations avec les organismes de transformation et de distribution

- L'occupation, la gestion et la valorisation des territoires concernés directement (le monde rural) et indirectement (les villes)

- L'évolution et la complexification des politiques publiques agricoles et rurales.

\section{Des changements de métier et d'approches du conseil}

En conséquence le métier d'agriculteur évolue et se diversifie, exigeant davantage de formation, de connaissances, mais aussi de savoir-faire à mobiliser, de capacité d'adaptation, ce qui implique également l'évolution des formations et des formateurs. Mais celui des chercheurs, des agents du Développement et des organismes d'accompagnement de l'agriculture se trouve également modifié dans ses démarches et dans les résultats obtenus ou attendus.

Trois changements progressifs me paraissent essentiels :

1. Le retour à l'importance de l'observation, dans le temps et l'espace, à la description des phénomènes ou des pratiques, sur le terrain, in situ, avec le concours des labo- ratoires et des modèles mathématiques, mais sans assujettissement à leurs seuls résultats. Du génome aux sols, aux climats, aux écosystèmes, aux produits, aux systèmes d'exploitation, aux territoires, aux comportements socio-économiques... les observatoires, les bases et banques de données, les typologies se développent (difficilement, mais leur nécessité s'impose). De l'agriculture raisonnée à l'agriculture biologique, l'observation et l'enregistrement par l'agriculteur prennent le pas sur l'application des préconisations techniques.

2. Le recours croissant à la démarche et à la modélisation systémique aux différents niveaux d'organisation, des systèmes biologiques aux systèmes socio-économiques.

3. La construction des questions et l'élaboration des solutions dans un cadre de partenariat interactif entre les différents acteurs concernés (de plus en nombreux et variés) dont les chercheurs et les agents de développement.

\section{De nouveaux objets et modes de Recherche-Développement}

Les évolutions technologiques s'inscrivent en partie et progressivement dans ce cadre nouveau qui s'avère plus exigeant et plus difficile que par le passé. De nouveaux objets doivent être considérés, tel que le territoire par exemple, et de nouvelles méthodes et techniques doivent être créés ou mobilisées pour les appréhender.

Je voudrais illustrer plus concrètement cet aspect à travers quelques exemples, choisis parmi des orientations que j'ai encadrées, allant des techniques de production jusqu'à la gestion des territoires, en passant par la satisfaction des consommateurs et des souhaits de fractions croissantes de la société.

- En matière de méthodes de production, des itinéraires techniques, des systèmes de 
culture et d'élevage, sont élaborés et assistés par des outils de pilotage, pour concilier la productivité, la qualité des produits, la protection de l'environnement, la qualité de vie sociale des exploitants agricoles et des ruraux. Ils permettent d'adapter les modes de production aux différents niveaux d'exigences affichés dans la voie de l'agriculture durable : de l'agriculture raisonnée, plus ou moins associée à l'agriculture de précision, aux changements de systèmes de production vers une plus grande durabilité, jusqu'à l'agriculture biologique qui a maintenant droit de cité, malgré les réticences des défenseurs du rationalisme. Les systèmes de culture plus extensifs, avec des pratiques de travail du sol renouvelées, la sélection de variétés de céréales « rustiques » mieux adaptées à ces systèmes, la production fruitière intégrée, les systèmes herbagers économes, le programme de R-D «porcherie verte » en sont quelques bons exemples, qui soulignent les évolutions techniques qui ont été ainsi engendrées.

Si les progrès des outils analytiques, informatiques et électroniques ou de la modélisation contribuent à ces évolutions, elles reposent souvent aussi sur l'expérience des agriculteurs et des éleveurs en recherche dans cette direction et sur l'observation de leurs pratiques. C'est avec eux que peuvent se construire les systèmes du futur ; les acquis des agriculteurs biologiques ont été la source de technologies redécouvertes ou nouvelles qui se généralisent ensuite.

- En matière de qualité des produits, les critères et méthodes de contrôle se sont multipliés, mobilisant un vaste arsenal d'outils analytiques permis par les avancées rapides des connaissances et des techniques. En outre on a mis en évidence les liens, existant mais mal connus, entre les facteurs et modes de production et les caractéristiques qualitatives des produits, resituant ainsi la part des technologies de transformation dans l'élaboration des qualités. Ce ne sont plus quelques techniques qui sont en cause mais l'ensemble des systèmes de production, de leur situation au sein des filières de produits et au sein des territoires de production. En travaillant en partenariat avec les producteurs de produits réputés, qualifiés par des AOC, des chercheurs ont renouvelé les démarches d'étude de la qualité, en partant du produit et en remontant jusqu'à la production aux techniques et aux savoirs mobilisés et à leurs interactions avec les milieux biophysiques et humains où ils sont mis en œuvre. Les relations entre les territoires, les systèmes et filières de production, les produits et les aspects sensoriels et culturels peuvent ainsi être explicitées et mieux maîtrisées. Or cela fait appel, par exemple, au progrès de la biologie moléculaire qui permet l'étude fine et la préservation des écosystèmes microbiens, sources d'arômes et de saveurs, plus ou moins liés à leur environnement local, et capables de limiter très fortement les risques sanitaires. L'organisation des filières de production et de leurs relations avec les territoires peut être abordée tant sur le plan technique que sur le plan économique et social et progresser. Les études économiques peuvent permettre de stimuler l'émergence de systèmes hybrides entre artisanat et industrie, capables de développer des stratégies de développement de ces produits de qualité. Les succès obtenus, par exemple dans les productions fromagères de zone de montagne, fruit d'un étroit partenariat avec les divers acteurs impliqués, soulignent la réalité et la pertinence de ces évolutions.

- Le bien-être animal est devenu une préoccupation notable de nos sociétés occidentales et la réponse aux attentes sociales en ce domaine implique aussi des évolutions technologiques. La prévention des maladies est une première étape indispensable (épidémiologie, vaccins, sélection de souches résistantes). Mais pour éliminer la souffrance banale et accroître le 
confort des animaux, en amélioration leur logement, leur alimentation et leur manipulation, les évolutions techniques reposent sur une meilleure connaissance de la physiologie et du comportement des animaux pour opérer les bons ajustements entre l'animal et son milieu, y compris par la sélection qui mobilise la génomique moderne. Mais il faut aussi accroître notre compréhension des relations entre l'éleveur et l'animal, dans le travail et la vie courante comme dans la perception du sens de son propre métier et du regard des autres. C'est en partant de ces éléments et de la compréhension des attentes des différentes catégories de citoyens vis-à-vis de cette question, que peuvent se construire les compromis nécessaires et les règles sociales qui en découlent pour concilier les évolutions technologiques de production, l'économie des filières et la satisfaction des attentes sociales.

- Concernant le travail dans les exploitations agricoles, il bénéficie des avancées en matière de mécanique, d'automatisation, de dispositifs électroniques d'alerte ou de régulation, d'informatique... Cela réduit généralement la pénibilité physique du travail, mais accroît le travail de bureau, le risque de panne et la tension nerveuse. Comme partout il est davantage fait appel aux facultés intellectuelles, mais pas forcément à l'initiative et à l'imagination. On progresse dans l'analyse du temps de travail et de son interférence avec les systèmes d'exploitation, pour aider à la prise de décision. Cependant il faut aussi prendre en compte les aspects psychologiques et sociologiques du métier qui se complexifie et se diversifie sans cesse, se combine à d'autres activités et est de plus en plus contraint par des règles et des travaux administratifs. Resituer, voire redéfinir, le sens de son métier est une des préoccupations majeures actuelles du monde agricole.

- La prise en compte de la gestion du territoire par l'agriculture, en relation avec les autres acteurs concernés, peut être bien illustrée par le renouveau du pastoralisme dans les zones de montagnes ou de parcours. S'appuyant en grande partie sur les pratiques et savoirs des bergers, les techniques pastorales ont été explicitées, améliorées tant au niveau de la conduite et de l'alimentation des troupeaux que de l'exploitation et du renouvellement des divers couverts végétaux. Cela a même entraîné de nouvelles conceptions sur la valeur alimentaire des divers végétaux offerts, et sur les capacités d'ingestion de ruminants, en interaction avec ces modes de conduite des surfaces et des animaux, redonnant de la valeur à des plantes et à des surfaces abandonnées. Ce pastoralisme rénové contribue à la fois à une production animale, souvent de grande qualité, à la lutte contre les incendies, l'érosion, la fermeture des paysages, à la vie locale et sa culture. Il implique un dialogue et des négociations entre les différents utilisateurs de ces territoires (forestiers, chasseurs, cueilleurs, promeneurs, aménageurs, résidents, élus...) qu'il est également possible de soutenir par des expertises scientifiques et des méthodes d'appui afin de parvenir à choisir les techniques les mieux adaptées à la conjonction des divers objectifs.

- C'est au niveau du développement territorial, régional ou local que la nécessité et la fécondité des démarches, pluridisciplinaires et en partenariat, de construction des questions et d'élaboration de solutions adaptées est particulièrement démonstrative. Mon expérience personnelle commencée sur le pays d'Aubrac dès 1965 , s'est poursuivie dans les Savoies, puis dans les programmes de recherche pour et sur le développement régional menés par la DADP de l'INRA dans 5 régions françaises. La réponse aux exigences sociétales traduites en questions de recherche et d'innovations souhaitables avec les partenaires des lieux, est à la base des avancées qui ont été réalisées, mobilisant les connaissances et les technologies et suscitant des innovations 
conceptuelles et opérationnelles. Les systèmes de production se sont effectivement transformés et adaptés en conservant leurs bases originelles. L'essor de zones difficiles, vouées par certains technocrates des administrations françaises ou européennes à l'abandon par l'agriculture, est la manifestation de l'intérêt de telles démarches collectives. Le développement de la contractualisation entre les acteurs concernés, au niveau des territoires comme des produits, constitue un des outils et aussi une des conséquences de ces évolutions. Les itinéraires techniques et les systèmes de production ont été de plus en plus finement définis dans ces conventions entre partenaires.

\section{Conclusions}

Les diverses technologies et leur évolution se trouvent donc mobilisées, revisitées, stimulées ou contestées par les attentes sociales actuelles. Leur prise en compte dans l'élaboration et l'évolution des systèmes d'exploitation reste essentielle mais comme élément qui n'est plus le principal déterminant de cette évolution. C'est dans les démarches actuelles, collectives et systémiques qu'elles se situent et trouvent une place plus ou moins forte.

Cependant dans la complexité et la prégnance croissante des cahiers des charges multiples auxquels doit adhérer l'exploitant agricole, qui résultent en grande partie de ces démarches collectives contractuelles, la marge de manœuvre de l'exploitant, les capacités d'innovations technologiques, ne se trouvent-elles pas de plus en plus réduites ? Comment de nouvelles technologies peuvent-elles émerger ? Le débat sur les OGM est symptomatique des difficultés d'ajustement entre technologies, systèmes de production et exigences sociétales ; s'il apparaît nécessaire de poursuivre les avancées considérables de la biologie moderne et de lui ouvrir diverses possibilités d'application aux bénéfices de la société tout entière, cela ne peut se faire que dans les limites d'un accord de la société sur les orientations, les précautions nécessaires et les applications acceptables, selon les systèmes de production.

Ces évolutions doivent donc s'inscrire dans celles des systèmes globaux qui comportent les attentes et les volontés des divers acteurs et impliquent dialogue et concertations. Pour dépasser les blocages inévitables, il faudrait davantage mobiliser et développer nos capacités de prospective, qui nécessitent la construction de visions communes des systèmes et l'élaboration de divers scénarios possibles, en tentant d'en évaluer les conséquences. Cette aide à l'élaboration de stratégies, proposables aux acteurs concernés et éclairées par leur participation à la réflexion, peut être un moyen de relancer ou de conforter le débat social et redonner des marges de manœuvre aux acteurs, notamment aux développeurs et aux chercheurs. 